\title{
Brain Imaging: A Promising Biomarker for Pain
}

\author{
Riccardo Serra*, Noah Leviton Gorelick, Betty Tyler and Michael Guarnieri \\ Department of Neurosurgery and Hunterian Neurosurgical Research Laboratory, Johns Hopkins University School of Medicine, USA
}

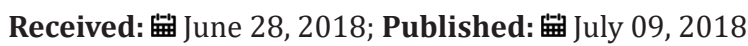

*Corresponding author: Riccardo Serra, Department of Neurosurgery and Hunterian Neurosurgical Research Laboratory, Johns Hopkins University School of Medicine, Baltimore, Maryland, USA

\begin{abstract}
Clinicians and researchers have the problem of objectively assessing the intensity and characteristics of pain. Furthermore, the widespread use of Visual-Analogue scales during the past decade highlights the need for a reliable and quantitative biological marker of pain. Mono- and multidimensional measures of pain, based on the activity of one or more brain regions involved in the quantification of painful inputs, have become the focus of extensive research. The growing availability of non-invasive functional imaging has in fact fueled a revolution in the field: a novel multiparametric pain signature was recently introduced and tested in patients, and proven effective in evaluating the intensity of painful inputs, and the effects of analgesics on those stimuli.

Abbrevations: VAS: Visual-Analogue Scales; CBF: Cerebral Blood Flow; ACC: Anterior Cingulate Cortex; IC: Insular Cortex; PFC: Prefrontal Cortex; BOLD: Blood-Oxygen-Level-Dependent; ASL: Arterial Spin Labeling; PET: Positron-Emission Tomography; EEG: electroencephalogram; DOT: Diffuse Optical Tomography; MEG: Magnetic Encephalography; CB: Cerebellum; FUS: Fusiform; HY: Hypothalamus; IFJ: Inferior Frontal Junction; MTG: Middle Temporal Gyrus; OG: Occipital Gyrus; PAG: Periaqueductal Gray Matter, PCC: Posterior Cingulate Cortex; SMA: Supplementary Motor Area; SMG: Supramarginal Gyrus; SPL: Superior Parietal Lobule; TG: Temporal Gyrus
\end{abstract}

\section{Introduction}

The recent growing interest in quantitative and reproducible biological markers for pain has prompted investigators to examine imaging techniques to detect and evaluate the cerebral representation of noxious stimuli[1,2]. Historically pain has been quantified with the help of Visual-Analogue Scales (VAS) that report the subjective experience as stated by the patient. The intrinsic variability and differing perceptive sensitivities in people unable to verbally communicate or among patients with disabilities have historically constituted a significant limitation to this tool[3,4].In recent years, MRI-based analysis of brain activation and local cerebral blood flow (CBF) has brought new hope to this field. By identifying the physiologic patterns of cortical/ subcortical activation, investigators are beginning to uncover the contribution of single areas to pain perception[5-7].The sensorydiscriminative, cognitive, and affective aspects of painful stimuli are in fact elaborated by six interconnected regions: the thalamus, the primary and secondary somatosensory cortices (SI-SII), the anterior cingulate cortex (ACC), the insular cortex (IC), and the prefrontal cortex (PFC)[8].

A variety of imaging techniques have been employed to study the changes in function and anatomy of these areas. Blood-oxygenlevel-dependent (BOLD) MRI contrast imaging is currently the most widespread method utilized, and its temporal resolution makes it a logical candidate to examine for acute pain syndromes $[9,10]$. Alternatively, arterial spin labeling (ASL) MRI techniques represent ongoing clinical pain and are employed as indicators of chronic pain and migraine[11,12]. Other approaches include diffusion tensor imaging (DTI) and structural MRI: the first provides information regarding the network that underlies pain management and perception[13], while the second uses voxel-base morphometry to quantify changes in white and grey matter[14,15]. Positronemission tomography (PET), electroencephalogram (EEG), diffuse optical tomography (DOT) and Magnetic Encephalography (MEG) have also been employed, but the high cost, low precision, and the necessity to shield the magnetic field limit their diffusion[16].

\section{The Brain Signature for Pain}

\section{Unidimensional measures of pain intensity}

Local changes in blood flow and cortical activity have been the focus of extensive research and a starting point for the characterization of the regions and nuclei involved in the elaboration and codification of pain. Among these, the SI-SII[17,18], the nucleus accumbens[19] and prefrontal regions[20], the ACC and posterior cingulate cortex[6], the amygdala[21], IC[22] and hippocampal formation[23] are theregions most commonly involved in the modulation of pain states, and their activity is strongly related to the intensity and location of the stimulus.BOLD and ASL MRI images have helped elucidate the role of each area in the physical and emotional aspects of pain. Multiple studies have shown that the frequency and pattern of activation of these regions, the amplitude of cortical signals, and their temporal relation to painful stimuli and heat are important clues to the identification of a partial pain 
signature that employs a limited number of areas[17,18]. PET, DOT, MEG and EEG have also provided an substantial data that may be used as indicators of pain modulation and intensity[24].

\section{Multidimensional Measures of Pain Intensity}

Although about $10 \%$ of the cerebral cortex seems to be associated with to pain processing[25], the presence of specific patterns of activation prompted scientists to elaborate a signature that encompasses multiple centers, better explaining the mechanism of pain representation and expression[26-28]. A more focused signal-to-noise ratio and higher specificity of imaging-mediated biological markers are allowed through the use of multiple inputs. This method may provide a solid starting point for future clinical uses of reliable measures of pain intensity, paving the road to algorithms for pain prediction and quantification. In 2013 Wager et al. first introduced a novel cerebral signature for pain based on the concomitant activity of a number of different cerebral regions. The thalamus, ACC, PFC, SII, cerebellum (CB), fusiform (FUS), hypothalamus (HY), inferior frontal junction (IFJ), middle temporal gyrus (MTG), occipital gyrus (OG), periaqueductal gray matter (PAG), posterior cingulate cortex (PCC), supplementary motor area (SMA), supramarginal gyrus (SMG), superior parietal lobule (SPL) and temporal gyrus (TG) were identified as the most important centers for pain elaboration, and therefore integrated in an algorithm that quantified the brain responses to painful inputs[29].

Furthermore, the concomitant and overlapping activation of multiple regions (bilateral anterior insula, medial thalamus, SII, and dorsal posterior insula) observed after exposure to stimuli of different intensities, provided a valid basis for the use of this algorithm for pain quantification, increasing its specificity and strength[29]. The signature response wasalso shown to be sensitive to the effects of analgesics with a reduction by $53 \%$ in signature intensity recorded after Remifentanil, an $\mu$-opioid agonist, administration [29].

The addition of artificial intelligence (AI) and machine learning to the pain algorithm will confer a higher reliability, sensitivity, and specificity to a signature that has extensively proven its efficacy, allowing for the identification of a larger number of fMRI patterns within the anatomic and functional pain circuitry[30].

\section{Future perspectives}

Modern non-invasive imaging techniques have profoundly changed the assessment and treatment of pain. These strategies are able to pinpoint individual differences in the central elaboration of painful inputs, quantifying the physiologic response to analgesics and supporting the development of novel therapeutics and treatment strategies[31]. The optimization of pain algorithms and signatures will likely allow a more accurate definition of pain experiences, reducing the importance of self-reports and their intrinsic unreliability. The advent of physiologically-based pain biomarkers will also reduce the risk of biases and improve the precision of measurements. fMRI techniques will therefore play a pivotal role in this process, setting a new standard in pain research and drug development.

\section{Conclusion}

Pain assessment is a complicated and not completely understood field. Investigators have spent decades searching for objective methods to establish pain levels in individuals with and without cognitive deficits. Only recently, after the introduction of various non-invasive imaging and electrophysiologic techniques, studies have clarified the role of brain areas whose function in pain management was not previously proven. Furthermore, the integration and concomitant use of measures of activity obtained from different regions of the Central Nervous System provide a reliable and specific tool for the identification of pain states, identifying new mechanisms of acute and chronic pain.

These instruments should therefore lead to the development of modern therapeutics that act on specific control systems, allowing researchers to accurately tailor the effects of drug candidates to the individual traits of each patient. The paradigm shift is revolutionary: novel molecules will target neurotransmitters' deficits, chronic changes in cortical function and gray matter volume, pain circuits and systems, improving the outcomes of patients with acute and chronic pain syndromes, and consequently their quality of life.

\section{References}

1. Borsook D, L Becerra, R Hargreaves (2011) Biomarkers for chronic pain and analgesia. Part 2: how, where, and what to look for using functional imaging. Discovery medicine 11(58): 209-219.

2. Davis KD, E Racine, B Collett (2012) Neuroethical issues related to the use of brain imaging: can we and should we use brain imaging as a biomarker to diagnose chronic pain? Pain153(8): 1555-1559.

3. Hjermstad MJ, Fayers PM, Haugen DF, Caraceni A, Hanks GW, et al. (2011) Studies comparing Numerical Rating Scales Verbal Rating Scales and Visual Analogue Scales for assessment of pain intensity in adults a systematic literature review. Journal of pain and symptom management 41(6): 1073-1093.

4. Price DD, Mc Grath PA, Rafii A, Buckingham B (1983) The validation of visual analogue scales as ratio scale measures for chronic and experimental pain. Pain 17(1): 45-56.

5. Borsook D, S Sava, L Becerra (2010) The pain imaging revolution: advancing pain into the 21st century. The Neuroscientist 16(2): 171185.

6. Apkarian AV, Bushnell MC, Treede RD, Zubieta JK (2005) Human brain mechanisms of pain perception and regulation in health and disease. European journal of pain 9(4): 463-463.

7. Apkarian AV, JA Hashmi, MN Baliki (2011) Pain and the brain: specificity and plasticity of the brain in clinical chronic pain. Pain152(3): S49-64.

8. Treede RD, Kenshalo DR, Gracely RH, Jones AK (1999) The cortical representation of pain. Pain 79(2-3): 105-111.

9. Ogawa S, Lee TM, Kay AR, Tank DW (1990) Brain magnetic resonance imaging with contrast dependent on blood oxygenation. Proceedings of the National Academy of Sciences 87(24): 9868- 9872.

10. Owen DG, Bureau Y, Thomas AW, Prato FS, St Lawrence KS (2008) Quantification of pain-induced changes in cerebral blood flow by perfusion MRI. Pain 136(1-2): 85-96.

11. Kato Y, Araki N, Matsuda H, Ito Y, Suzuki C (2010) Arterial spin-labeled MRI study of migraine attacks treated with rizatriptan. The journal of headache and pain 11(3): 255-258.

12. Wasan AD, Loggia ML, Chen LQ, Napadow V, Kong J, et al. (2011) Neural correlates of chronic low back pain measured by arterial spin labeling. Anesthesiology: The Journal of the American Society of Anesthesiologists 115(2): 364-374. 
13. Conturo TE, Lori NF, Cull TS, Akbudak E, Snyder AZ, et al. (1999) Tracking neuronal fiber pathways in the living human brain. Proceedings of the National Academy of Sciences 96(18): 10422-10427.

14. Smith SM, Jenkinson M, Johansen-Berg H, Rueckert D, Nichols TE, et al. (2006) Tract-based spatial statistics: voxel wise analysis of multi-subject diffusion data. Neuroimage 31(4): 1487-1505.

15. Davis KD, Moayedi M (2013) Central mechanisms of pain revealed through functional and structural MRI. Journal of Neuroimmune Pharmacology 8(3): 518-534.

16. Morton DL, Sandhu JS, Jones AK (2016) Brain imaging of pain: state of the art. Journal of pain research 9: 613-624.

17. Chen JI, Ha B, Bushnell MC, Pike B, Duncan GH (2002) Differentiating noxious-and innocuous-related activation of human somatosensory cortices using temporal analysis of fMRI. Journal of neurophysiology 88(1): 464-474.

18. Upadhyay J, Pendse G, Anderson J, Schwarz AJ, Baumgartner R, et al. (2010) Improved characterization of BOLD responses for evoked sensory stimuli. Neuroimage 49(3): 2275-2286.

19. Baliki MN, Geha PY, Fields HL, Apkarian AV (2010) Predicting value of pain and analgesia: nucleus accumbens response to noxious stimuli changes in the presence of chronic pain. Neuron 66(1): 149- 160.

20. Baliki MN, Chialvo DR, Geha PY, Levy RM, Harden RN, Parrish TB, et al. (2007) Chronic Pain and the Emotional Brain: Specific Brain Activity Associated with Spontaneous Fluctuations of Intensity of Chronic Back Pain. Headache 26(47): 12165-12173.

21. Neugebauer V, Li W, Bird GC, Han JS (2004) The amygdala and persistent pain. The Neuroscientist 10(3): 221-234.

22. Ostrowsky K, Magnin M, Ryvlin P, Isnard J, Guenot M, et al. (2002) Representation of pain and somatic sensation in the human insula: a study of responses to direct electrical cortical stimulation. Cerebral Cortex 12(4): 376-385.

23. Liu MG, Chen J (2009) Roles of the hippocampal formation in pain information processing. Neuroscience bulletin 25(5): 237-266.

24. Becerra L, Harris W, Joseph D, Huppert T, Boas DA, et al. (2008) Diffuse optical tomography of pain and tactile stimulation: activation in cortical sensory and emotional systems. Neuroimage 41(2): 252-259.

25. Yarkoni T, Russell A Poldrack, Thomas E Nichols, David C Van Essen (2011) Large-scale automated synthesis of human functional neuroimaging data. Nature methods 8(8): 665.

26. Garcia Larrea L (2012) Insights gained into pain processing from patients with focal brain lesions. Neuroscience letters 520(2): 188-191.

27. Chen LM, Dillenburger BC, Wang F, Tang CH (2012) Differential fMRI activation to noxious heat and tactile stimuli in parasylvian areas of new world monkeys. Pain 153(1): 158-169.

28. Vierck CJ, Whitsel BL, Favorov OV, Brown AW, Tommerdahl M (2013) Role of primary somatosensory cortex in the coding of pain. PAIN $®$ 154(3): 334-344

29. Wager TD, Lauren Y Atlas, Martin A Lindquist, Mathieu Roy, Choong-Wan Woo, et al. (2013) An fMRI-based neurologic signature of physical pain. New England Journal of Medicine 368(15): 1388-1397.

30. Kamitani Y, Tong F (2005) Decoding the visual and subjective contents of the human brain. Nature neuroscience 8(5): 679-685.

31. Christopher de Charms R, Maeda F, Glover GH, Ludlow D, Pauly JM, et al. (2005) Control over brain activation and pain learned by using real-time functional MRI. Proceedings of the National Academy of Sciences of the United States of America 102(51): 18626-18631.
ISSN: 2574-1241

DOI: 10.26717/BJSTR.2018.06.001369

Riccardo Serra. Biomed J Sci \& Tech Res

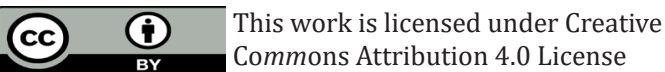

Submission Link: https://biomedres.us/submit-manuscript.php

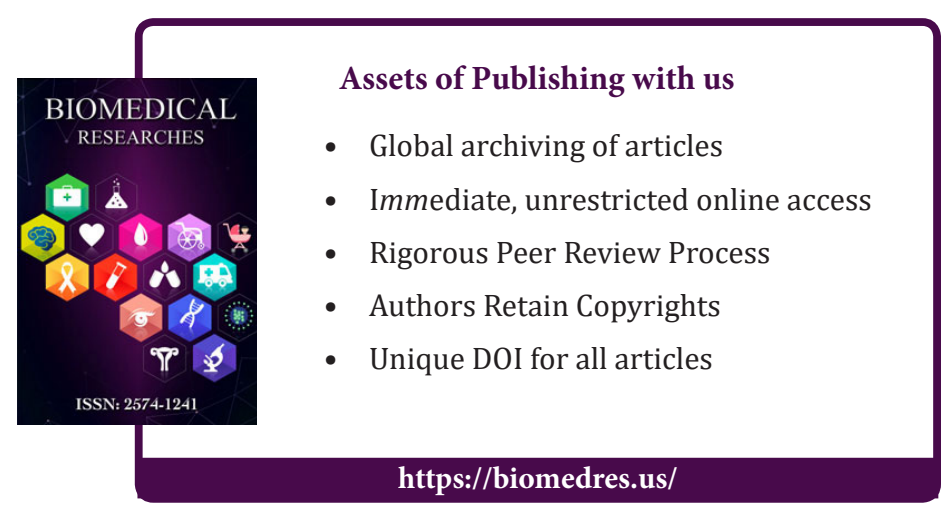

